\title{
Microstructured organic ferroelectric thin film capacitors by solution micromolding
}

\author{
Thomas Lenz, Dong Zhao, George Richardson, Ilias Katsouras, Kamal Asadi, Gunnar \\ Glasser, Samuel T. Zimmermann, Natalie Stingelin, W. S. Christian Roelofs, Martijn \\ Kemerink, Paul W. M. Blom and Dago M. de Leeuw
}

\section{Linköping University Post Print}

\section{Tweet}

N.B.: When citing this work, cite the original article.

Original Publication:

Thomas Lenz, Dong Zhao, George Richardson, Ilias Katsouras, Kamal Asadi, Gunnar Glasser, Samuel T. Zimmermann, Natalie Stingelin, W. S. Christian Roelofs, Martijn Kemerink, Paul W. M. Blom and Dago M. de Leeuw, Microstructured organic ferroelectric thin film capacitors by solution micromolding, 2015, Physica Status Solidi (a) applications and materials science, (212), 10, 2124-2132.

http://dx.doi.org/10.1002/pssa.201532267

Copyright: Wiley-VCH Verlag

http://www.wiley-vch.de/publish/en/

Postprint available at: Linköping University Electronic Press

http://urn.kb.se/resolve?urn=urn:nbn:se:liu:diva-122535 


\section{Microstructured organic ferroelectric thin film capacitors by solution micromolding}

Thomas Lenz ${ }^{1,2}$, Dong Zhao ${ }^{1}$ (赵冬), George Richardson ${ }^{3}$, Ilias Katsouras ${ }^{1,4}$, Kamal Asadi ${ }^{1,5}$, Gunnar Glaßer ${ }^{1}$, , Samuel T. Zimmermann ${ }^{3}$, Natalie Stingelin ${ }^{3}$, W.S. Christian Roelofs ${ }^{6}$, Martijn Kemerink $^{6,7}$, Paul W.M. Blom ${ }^{1,2}$ and Dago M. de Leeuw ${ }^{1,8^{*}}$

${ }^{1}$ Max-Planck Institute for Polymer Research, Ackermannweg 10, 55128 Mainz, Germany

${ }^{2}$ Graduate School Materials Science in Mainz, Staudinger Weg 9, 55128 Mainz, Germany

${ }^{3}$ Department of Materials and Centre of Plastic Electronics, Imperial College London, London $S W 72 A Z, U K$

${ }^{4}$ Holst Centre, High Tech Campus 31,5656AE Eindhoven, The Netherlands

${ }^{5}$ Max Planck Graduate Center, Staudinger Weg 9, 55128 Mainz, Germany

${ }^{6}$ Department of Applied Physics, Eindhoven University of Technology, P.O. Box 513, 5600 MB Eindhoven, The Netherlands

${ }^{7}$ Complex Materials and Devices, Department of Physics, Chemistry and Biology, Linköping University, 58183 Linköping, Sweden

${ }^{8}$ King Abdulaziz University, Abdullah Sulayman, 22254 Jeddah, Saudi Arabia

[*] E-mail: deleeuw @mpip-mainz.mpg.de

Keywords: ferroelectric materials, $\mathrm{P}(\mathrm{VDF}-\mathrm{TrFE})$, solution micromolding, thin film capacitor, Sawyer-Tower, shunt measurements 


\section{Abstract}

Ferroelectric nanostructures offer a promising route for novel integrated electronic devices such as non-volatile memories. Here we present a facile fabrication route for ferroelectric capacitors comprising a linear array of the ferroelectric random copolymer of vinylidenefluoride and trifluoroethylene ( $\mathrm{P}(\mathrm{VDF}-\mathrm{TrFE})$ ) interdigitated with the electrically insulating polymer polyvinyl alcohol (PVA). Micrometer size line gratings of both polymers were fabricated over large area by solution micromolding, a soft lithography method. The binary linear arrays were realized by backfilling with the second polymer. We investigated in detail the device physics. The electrical equivalent circuit is a linear capacitor of PVA in parallel with a ferroelectric capacitor of P(VDF$\mathrm{TrFE})$. The binary arrays are electrically characterized by both conventional Sawyer-Tower and

shunt measurements. The dependence of the remanent polarization on the array topography is explained by numerical simulation of the electric field distribution. 


\section{Introduction}

Ferroelectric materials are emerging in organic electronics as attractive candidates for data storage applications, i.e. non-volatile memory elements that retain their data when the power is turned off, and that furthermore can be read, programmed and erased electrically. The low processing temperature allows device fabrication on foils, thereby enabling flexible electronics. The most commonly used organic ferroelectric materials are poly(vinylidenefluoride) (PVDF) and its random copolymers with trifluoroethylene ( $\mathrm{P}(\mathrm{VDF}-\mathrm{TrFE}))$. The chemical structure is presented in Fig. 1b. In comparison to other organic ferroelectrics, the copolymer P(VDF-TrFE) exhibits advantageous properties. It can be processed from solution and comprising devices exhibit a relatively large remnant polarization, low leakage current and short switching times of 1 $\mu \mathrm{s} .^{1}$

For high density data storage the ferroelectric polymer needs to be patterned with small feature sizes. However, photolithography and developing of the exposed patterns can compromise the structural integrity and deteriorate the ferroelectric properties of the polymer. Therefore, alternative patterning technologies are being investigated. For instance P(VDF-TrFE) films have been patterned by ink jet printing, ${ }^{2,3}$ photo-crosslinking upon adding a bisazide photoinitiator, ${ }^{4}$ microimprinting, ${ }^{5,6}$ nanoimprint lithography ${ }^{7}$ and nano-embossing. ${ }^{8,9}$ A review on patterned piezo-, pyro- and ferroelectric polymers has been reported in $2010 .^{10}$ In recent years soft lithography has emerged as a low-cost, high-throughput patterning technique, ideally suited to pattern polymeric materials. ${ }^{11}$ An elastomeric stamp with a network of interconnected channels is made of poly(dimethylsiloxane) (PDMS). The relief features on its surface can be replicated by pressing the stamp into a polymer film or by putting the stamp into intimate contact with the substrate followed by spontaneously filling of the channels by capillary forces (MIMIC). ${ }^{12}$ The stamp is subsequently removed and the microstructures, in a pattern complimentary to that present in the stamp, are replicated on the surface. PVDF micropatterns have been fabricated both by microimprinting ${ }^{5,6,13}$ and capillary micromolding. ${ }^{13,14}$

The ferroelectric and piezoelectric properties have been reported to depend on the length scale. PVDF and $\mathrm{P}(\mathrm{VDF}-\mathrm{TrFE})$ nanowires have been prepared in mesoporous silica and porous 
alumina. The melting and crystallization temperature decrease with decreasing pore diameter. ${ }^{15}$ The Curie temperature is not affected. ${ }^{15,16}$ For $\mathrm{P}(\mathrm{VDF}-\mathrm{TrFE})$ an increase of the piezoelectric coefficient has been reported. ${ }^{17,18}$ The enhanced crystallization upon confinement ${ }^{19,20}$ is reflected in the lowering of the coercive field down to $10 \mathrm{MV} / \mathrm{m},{ }^{8,18,21}$ as compared to $60 \mathrm{MV} / \mathrm{m}$ typically measured for standard, unpatterned, thin films. P(VDF-TrFE) has been confined within selfassembled organosilicate lamellae and used as gate dielectric in organic field-effect transistors. ${ }^{22}$ Low voltage operation was achieved by virtue of the low value of the coercive field of the confined $\mathrm{P}(\mathrm{VDF}-\mathrm{TrFE})$.

Patterned ferroelectrics can lead to new device architectures. Memory diodes have been reported that were based on phase separated blends of $\mathrm{P}(\mathrm{VDF}-\mathrm{TrFE})$ and organic semiconducting polymers. ${ }^{23-26}$ The structure of these blends consists of semiconducting columns in a ferroelectric matrix. The bi-stable polarization state of the ferroelectric yields the binary information that can non-destructively be read out by current through the semiconducting columns. The operation is based on the modulation of the injection barrier by the capacitive stray field at the interface between the ferroelectric and the semiconductor. ${ }^{27}$

However, phase separation may lead to an irregular and broad distribution of semiconducting column widths. There is a direct need for alternative techniques to pattern the ferroelectric polymer. In order to obtain more regular structures diodes were fabricated by nanomolding the $\mathrm{P}(\mathrm{VDF}-\mathrm{TrFE})$ followed by filling the resulting open spaces with a semiconductor ${ }^{28,29}$ or by surface directed phase separation. ${ }^{30}$

Reports on the physics of devices comprising patterned organic ferroelectric materials are however limited. Ferroelectric properties are typically investigated locally by e.g. piezo-response force microscopy, ${ }^{7,8,13,16,21,27}$ but scarcely in actual devices. ${ }^{17,18,21-23,27,31,32}$ Careful analysis is required, as both the characterization method and the device geometry can influence the measured ferroelectric characteristics. Here we investigate for the first time the device physics of capacitors based on linear gratings of $\mathrm{P}(\mathrm{VDF}-\mathrm{TrFE})$. The space between the ferroelectric lines is backfilled with an insulting polymer yielding a binary linear polymer array. The consequences of 
the resulting equivalent circuit on the electrical characterization by Sawyer-Tower measurements are analyzed and experimentally verified.

Specifically, here we fabricate gratings of $\mathrm{P}(\mathrm{VDF}-\mathrm{TrFE})$ by solution micromolding as schematically depicted in Fig. 1a. We systematically varied the concentration of P(VDF-TrFE) in solution and characterized the resulting microstructure and topography by atomic force microscopy (AFM) and scanning electron microscopy (SEM).

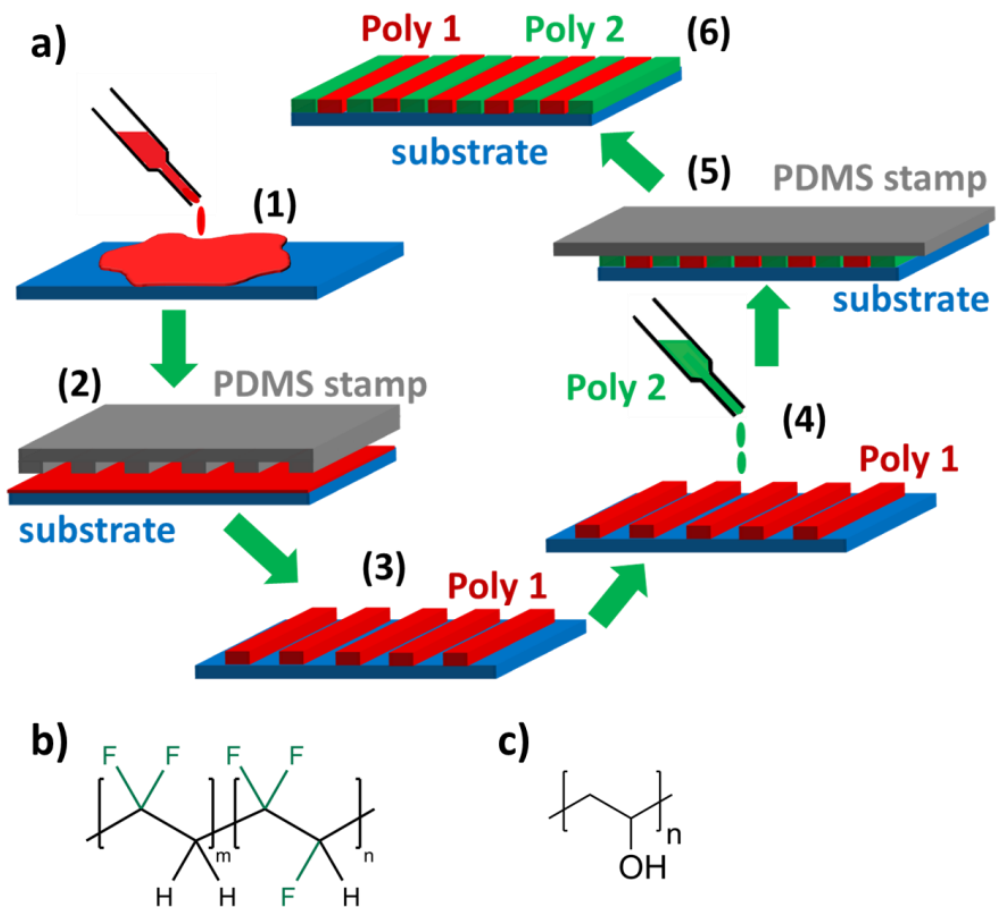

Fig. 1. Solution micromolding. (a) Fabrication of a binary linear polymer array by solution micromolding. The solution of a polymer is poured onto the substrate and a PDMS stamp with relief structures on its surface is pressed onto the substrate surface using a hot press. After the solvent has fully evaporated, the stamp is removed and a complementary linear grating of the polymer is obtained. The space in between the lines can then be backfilled with a second polymer by hot pressing with a flat stamp, leading to a binary linear array of two polymers. $(b)$ Chemical structure of $P(V D F-T r F E)$ and (c) PVA. 
To allow the fabrication of discrete capacitors and to measure the ferroelectric properties, the space between the $\mathrm{P}(\mathrm{VDF}-\mathrm{TrFE})$ lines has to be backfilled with an electrically insulating polymer. Polymer re-dissolution and stamp swelling have to be avoided. ${ }^{14}$ We therefore chose polyvinyl alcohol (PVA, chemical structure in Fig. 1c) as the insulating polymer, since its solvent, deionized water, neither swells the PDMS stamp nor dissolves P(VDF-TrFE). ${ }^{33}$ To complete the study, we also fabricated capacitors in which PVA was patterned first, followed by backfilling with $\mathrm{P}(\mathrm{VDF}-\mathrm{TrFE})$. All binary arrays exhibit a non-planar topography, since the backfilling inherently leads to lower feature heights for the second, backfilled polymer. We show that the measured ferroelectric properties, such as the apparent remanent polarization, depend on the details of the topography, which we explain by numerical simulations of the electric field distribution. The developed technology and characterization protocol may allow to study the effect of confinement on the ferroelectric properties of organic materials by scaling down the lateral dimensions of the grating.

\section{Experimental}

All chemicals were used as received. The random copolymer P(VDF-TrFE) (65\%-35\%) was purchased from Solvay. The number- and weight-average molecular weight, $\mathrm{Mn}$ and $\mathrm{Mw}$, were measured with gel permeation chromatography (GPC) versus polystyrene standards and amounted to $147 \mathrm{~kg} / \mathrm{mol}$ and $296 \mathrm{~kg} / \mathrm{mol}$, respectively. The polydispersity amounted to 2.01 . The weight average molecular weight of polyvinyl alcohol (PVA) (Sigma Aldrich) was 80-90 kg/mol. Dimethyl sulfoxide (DMSO) and methyl ethyl ketone (MEK) were used as solvents for P(VDFTrFE), PVA was dissolved in deionized water.

A 4" silicon master with anti-sticking coating was obtained from NIL Technology (Denmark) and used as received. It provides a periodic line grating with pitch size of $4 \mu \mathrm{m}$. The line width is 1.3 $\mu \mathrm{m}$ and the height is $2 \mu \mathrm{m}$. Stamps complementary to the master grating were prepared from polydimethylsiloxane (PDMS). The stamp was fabricated using Sylgard 184 silicone elastomer mixed with a curing agent (Dow Corning, USA) in a ratio of 10:1 by weight. The silicone elastomer mixture was then cast on the master. The stamp/master assembly was evacuated in a 
desiccator in order to detrap air bubbles. After curing for 3 hours at $60{ }^{\circ} \mathrm{C}$, the PDMS stamp was peeled off from the master.

As schematically depicted in Fig. 1a, polymer gratings were fabricated on glass substrates with bottom electrodes of $100 \mathrm{~nm}$ thick $\mathrm{Au}$ with a $5 \mathrm{~nm} \mathrm{Cr}$ adhesion layer, prepared by thermal evaporation through a shadow mask. The substrates were subsequently cleaned with UV/ozone and a drop of the polymer solution, $\mathrm{P}(\mathrm{VDF}-\mathrm{TrFE})$ in DMSO or PVA in deionized water, was put onto the substrate. The PDMS stamp was positioned on top and the substrate/stamp assembly was hot-pressed for 2 hours (hot-press from Paul-Otto Weber GmbH, Germany). For PVA the temperature was set to $70{ }^{\circ} \mathrm{C}$ while for $\mathrm{P}(\mathrm{VDF}-\mathrm{TrFE})$ the temperature was set to $140{ }^{\circ} \mathrm{C}$, which is above the Curie temperature $\left(120{ }^{\circ} \mathrm{C}\right)$ but below the melting temperature $\left(150{ }^{\circ} \mathrm{C}\right)$ of the copolymer.

Phase identification of $\mathrm{P}(\mathrm{VDF}-\mathrm{TrFE})$ was performed with specular wide angle X-ray diffraction measurements, using a Philips PW1820 diffractometer with $\mathrm{Cu}$ radiation. The peak at a wave vector of $1.3 \AA^{-1}\left(2 \Theta\right.$ of $\left.19.7^{\circ}\right)$ is characteristic for the combined $(110) /(200)$ diffraction of the monoclinic low temperature phase of $\mathrm{P}(\mathrm{VDF}-\mathrm{TrFE})$.

In order to electrically characterize the gratings, the empty space between the lines was backfilled with an electrically insulating second polymer, before depositing the top electrode. For P(VDFTrFE) gratings, a drop of PVA solution was put onto the substrate and a flat, unstructured PDMS stamp was pressed onto it, again utilizing the hot-press at $70{ }^{\circ} \mathrm{C}$ for 2 hours. In the inverse process, PVA gratings were equivalently backfilled with $\mathrm{P}(\mathrm{VDF}-\mathrm{TrFE})$ at $140{ }^{\circ} \mathrm{C}$. Here, $\mathrm{P}(\mathrm{VDF}-$ TrFE) was dissolved in methyl ethyl ketone (MEK) instead of DMSO, because the latter would compromise the integrity of the PVA grating.

Capacitor fabrication was finished by evaporation of $100 \mathrm{~nm} \mathrm{Au}$ as top electrode through a shadow mask. This provides a crossbar capacitor array, in which each discrete capacitor has an area of about $0.1 \mathrm{~mm}^{2}$. For comparison, benchmark capacitors were fabricated using unpatterned $\mathrm{P}(\mathrm{VDF}-\mathrm{TrFE})$ thin films with a thickness of about $0.5 \mu \mathrm{m}$, obtained by spin-coating from MEK, followed by an annealing step at $140{ }^{\circ} \mathrm{C}$ for 2 hours to enhance the crystallinity. 
The thickness of the films was measured with a surface profilometer. The topography and the morphology were investigated with Atomic Force Microscopy (AFM) (Nanoscope Dimension 3100, Bruker). Furthermore, the gratings were imaged as delivered with low voltage Scanning Electron Microscopy (LVSEM) using a Zeiss 1530 Gemini. Energy dispersive X-ray (EDX) spectroscopy was performed with a Hitachi SU8000 SEM in combination with a Bruker XFlash5010 Silicon Drift Detector. Micrographs for imaging were acquired at accelerating voltages up to $1 \mathrm{kV}$. Higher voltages could be applied after coating with $5 \mathrm{~nm}$ of sputtered Pt.

Ferroelectric hysteresis loops were extracted both from Sawyer-Tower and shunt measurements, using a Tektronix AFG3022B function generator, a LeCroy waverunner LT372 oscilloscope and a Krohn-Hite $7602 \mathrm{M}$ wide-band amplifier. The frequency was set at $100 \mathrm{~Hz}$. Impedance was measured using a Schlumberger Si 1260 Impedance Analyzer. All electrical measurements were performed in a vacuum probe station pressurized at $10^{-6} \mathrm{mbar}$.

\section{Fabrication of binary linear arrays}

Fabrication of linear gratings by capillary micromolding inevitably led to incomplete filling of the channels in the PDMS stamp. The replicated lines were not continuous, in agreement with literature reports. ${ }^{14}$ To circumvent the formation of line defects we focused on solution micromolding, as schematically depicted in Fig. 1a. We systematically varied the concentration of $\mathrm{P}(\mathrm{VDF}-\mathrm{TrFE})$. SEM micrographs of the replicated gratings are presented in Fig. 2. At a high concentration of $300 \mathrm{mg} / \mathrm{ml}$, an almost complete filling of the $2 \mu \mathrm{m}$ deep channels was obtained. However, due to the high viscosity of the concentrated solution, a thin P(VDF-TrFE) film is left in between the lines, the presence of which can interfere with the electrical characterization. The residual film is not present at low concentrations, however below $100 \mathrm{mg} / \mathrm{ml}$ the filling is incomplete and replicated lines are discontinuous. The optimum concentration is around 150 $\mathrm{mg} / \mathrm{ml}$. The lines are then continuous and the debris between the lines is minimal. The height of the patterns is about $1 \mu \mathrm{m}$, which is the maximum thickness for convenient electrical characterization. 


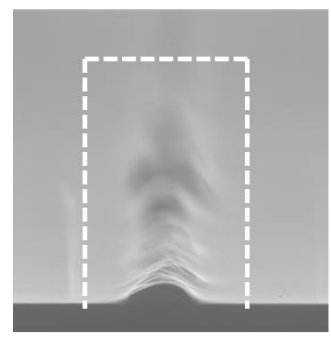

$10 \mathrm{mg} / \mathrm{ml}$

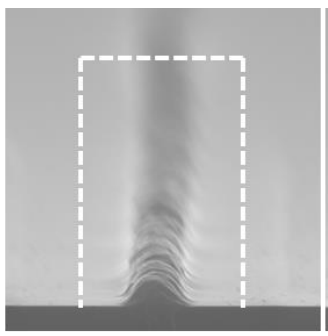

$50 \mathrm{mg} / \mathrm{ml}$

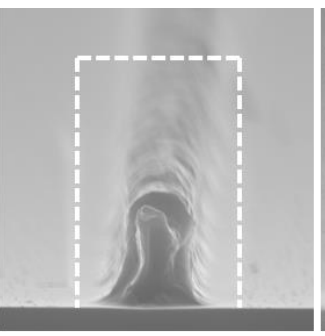

$100 \mathrm{mg} / \mathrm{ml}$

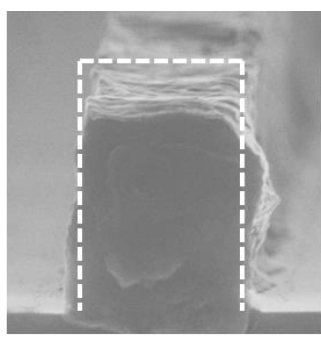

$200 \mathrm{mg} / \mathrm{ml}$

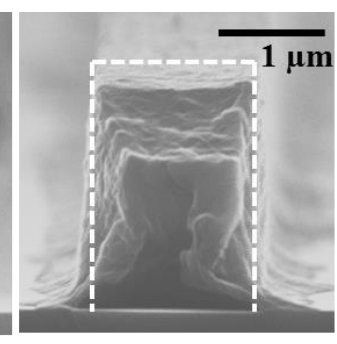

$300 \mathrm{mg} / \mathrm{ml}$ concentration of $P(V D F-T r F E)$ in DMSO

Fig. 2. Fill factor of the relief pattern. SEM micrographs of micromolded $P(V D F-T r F E)$ lines fabricated with different concentrations of $P(V D F-T r F E)$ in DMSO. The dotted line represents the shape of the capillary in the stamp.

The morphology and topography were investigated with SEM and AFM. Typical images of micromolded gratings are presented in Fig. 3a,c respectively. For comparison, SEM and AFM images taken from a spin-coated, and subsequently annealed, $\mathrm{P}(\mathrm{VDF}-\mathrm{TrFE})$ film are presented in Fig. 3b,d. The SEM images show the needle-like morphology characteristic for crystalline $\mathrm{P}(\mathrm{VDF}-\mathrm{TrFE}) .{ }^{34-36}$ The size of the crystals in the micromolded grating is much larger than for the spin-coated film. The origin is presumably Ostwald ripening; the coarsening originates from the presence of solvent in the stamp at elevated temperature. Specular XRD measurements have shown that the needles consist of the ferroelectric low temperature phase of $\mathrm{P}(\mathrm{VDF}-\mathrm{TrFE})$. The microstructural differences between spin-coated and micromolded $\mathrm{P}(\mathrm{VDF}-\mathrm{TrFE})$ is confirmed by AFM measurements. The P(VDF-TrFE) lines consist of large needle-like crystals in agreement with literature reports. ${ }^{34,35,37}$ The lines are dense and continuous; there is hardly any debris in between the lines. 
a)

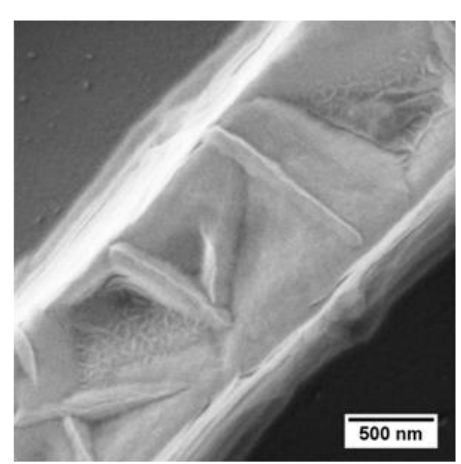

c)

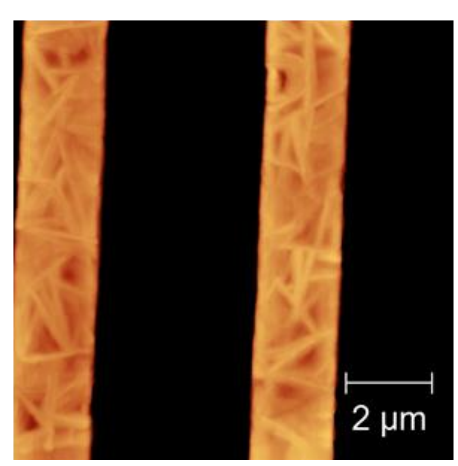

b)

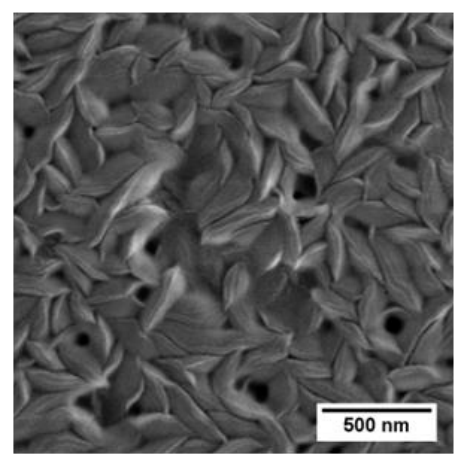

d)

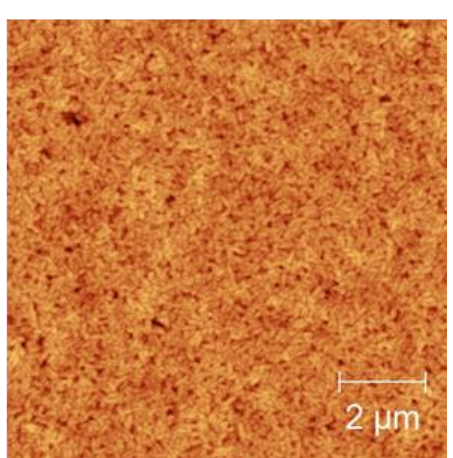

Fig. 3. Morphology of $\mathbf{P}(\mathrm{VDF}-\mathrm{TrFE})$. SEM micrographs of (a) a micromolded grating and (b) a spin-coated P(VDF-TrFE) thin film. AFM height images of (c) a mircomolded grating (colour scale ranges from $700 \mathrm{~nm}$ to $1 \mu \mathrm{m})$ and (d) a spin-coated $P(V D F-T r F E)$ thin film (colour scale ranges from $0 \mathrm{~nm}$ to $100 \mathrm{~nm}$ ). We adapted the colour scales to emphasize the top morphology.

To measure the ferroelectric properties of the gratings, we backfilled the gaps with PVA as an electrically insulating polymer. The filling increases with the concentration of the PVA solution. However, at very high concentration the viscosity is so high that the PVA overflows the P(VDFTrFE) gratings. As a compromise between filling and processability we used $500 \mathrm{mg} / \mathrm{ml}$. Fig. 4a,b show SEM micrographs before and after backfilling. The cross sections were obtained by mechanically breaking the substrate. Fig. 4a shows the P(VDF-TrFE) line grating. As discussed above the lines are continuous, dense and uniform, and there is hardly any debris in between. The cross section after backfilling is presented in Fig. $4 \mathrm{~b}$. There is a conformal thin gold film on top, as the micrograph was obtained from a finished capacitor. The image of the binary array shows topography as the height of the backfilled PVA features is lower than that of the original P(VDF- 
TrFE) grating. The initial micromolded grating always protrudes in the height profile of the binary array. The aspect ratio of the array is about 1:2.

To confirm that PVA is perfectly interdigitated with the P(VDF-TrFE) grating, we performed element mapping by Energy-dispersive X-ray spectroscopy (EDX). The top view SEM micrograph is presented in Fig. 4c and the corresponding EDX map is presented in Fig. 4d. The presence of $\mathrm{F}$ atoms is indicated in blue and of $\mathrm{O}$ atoms in green. PVA is confined between the $\mathrm{P}(\mathrm{VDF}-\mathrm{TrFE})$ lines; there is hardly any PVA on top of P(VDF-TrFE). This can be expected from the large difference in polarity between these two materials. The absence of PVA on top of the $\mathrm{P}(\mathrm{VDF}-\mathrm{TrFE})$ is further substantiated by thickness measurements before and after backfilling and by AFM measurements that show the characteristic needle like morphology of P(VDF-TrFE).

a)

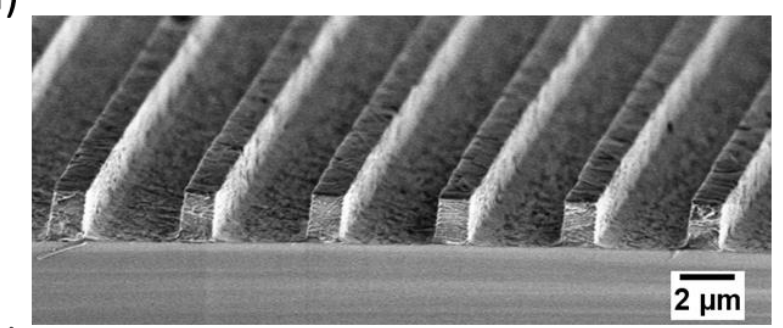

b)

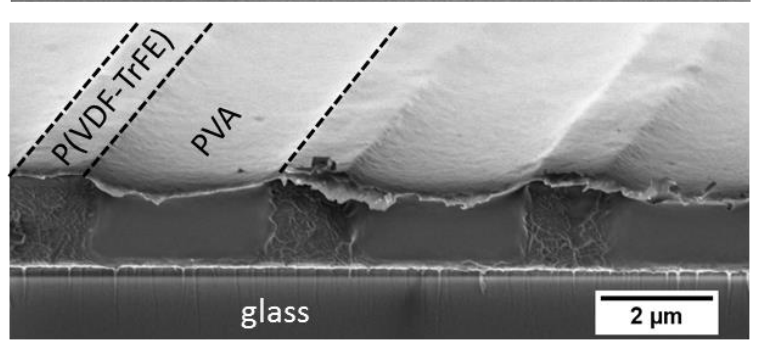

c)

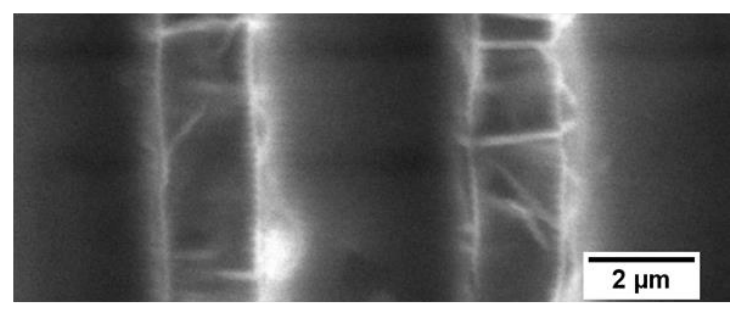

d)

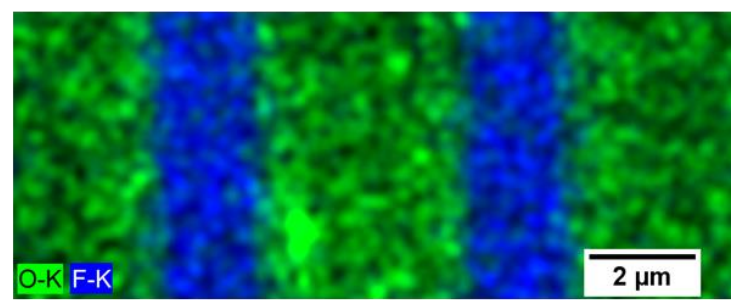

Fig. 4. Micromolded linear arrays of P(VDF-TrFE) and PVA. (a) SEM micrographs of a cross section of (a) a P(VDF-TrFE) line grating and (b) a backfilled array. A conformal thin Au film can be seen on top and below the array, as the micrograph is taken from a finished capacitor. (c) SEM top view image and (d) corresponding EDX map of the backfilled array. The distribution of $F$ and $O$ atoms is presented in blue and green respectively. 
Solution micromolding is generic in the creation of linear arrays. To that end, we fabricated inverted arrays. We first prepared a line grating of PVA, followed by backfilling with P(VDFTrFE). The AFM height profiles of the grating and binary array are presented in Fig. 5a,c. Lighter colors indicate higher features. The P(VDF-TrFE) lines show large crystals, similar as in Fig. 3. Typical line profiles are presented in Fig. 5b. The inverted array also exhibits a non-planar topography. The height of the backfilled P(VDF-TrFE) is lower than that of the micromolded PVA; the aspect ratio is about 1:2. For all binary arrays the initial micromolded grating protrudes in the height profile. As illustrated in Figs. $4 \mathrm{~b}$ and $5 \mathrm{~b}$ the surface of the backfilled polymer is concave, because upon backfilling the second polymer also covers the sidewalls of the previously patterned grating.


Fig.5. Inverted linear array. AFM height images of (a) a PVA micromolded grating (colour scale ranges from $0 \mathrm{~nm}$ to $50 \mathrm{~nm}$ to get an optimum picture of the grating) and (c) the corresponding binary linear array (colour scale ranges from $0 \mu \mathrm{m}$ to $1.2 \mu \mathrm{m}$ ) after backfilling with P(VDF-TrFE). (b) Typical line profiles for the two AFM micrographs. Note that the position of the red line (after backfilling) was adapted to better illustrate the change of topography upon backfilling.

\section{Ferroelectric characterization of microstructured capacitors}

Typical ferroelectric hysteresis loops for the binary linear arrays, as measured in a standard Sawyer-Tower system, are presented in Fig. 6, where the displacement is presented as a function of electric field. For comparison we included the hysteresis loop for an unpatterned P(VDF- 
TrFE) film (black curve). This benchmark capacitor exhibits a coercive field of about $55 \mathrm{MV} / \mathrm{m}$ and a remanent polarization of $70 \mathrm{mC} / \mathrm{m}^{2}$, in good agreement with literature values. ${ }^{34,35}$ The blue curve is a typical measurement for a binary linear array consisting of a micromolded PVA grating backfilled with $\mathrm{P}(\mathrm{VDF}-\mathrm{TrFE})$. The hysteresis loop is comparable to that of the unpatterned benchmark capacitor, exhibiting a similar remanent polarization. The coercive field is slightly higher, probably because, as a convention, it was calculated using the minimum thickness in the concave thickness profile.

The orange curve is a typical measurement for a linear array consisting of a micromolded $\mathrm{P}(\mathrm{VDF}-\mathrm{TrFE})$ grating backfilled with PVA. In this case, the hysteresis loop is significantly different from that of the unpatterned, benchmark capacitor. The remanent polarization is considerably lower, viz. around $40 \mathrm{mC} / \mathrm{cm}^{2}$. Furthermore, the slope of displacement versus electric field at zero bias is much steeper, which indicates that the linear dielectric capacitance is much higher. To exclude depolarization as the origin of the high capacitance, we measured the inner hysteresis loops at applied fields below the coercive field. As shown in table 1, the capacitance obtained from the inner hysteresis loops is similar to the capacitance that can be extracted by small signal impedance measurements, and significantly higher than the calculated geometrical capacitance. The origin is not yet fully understood.

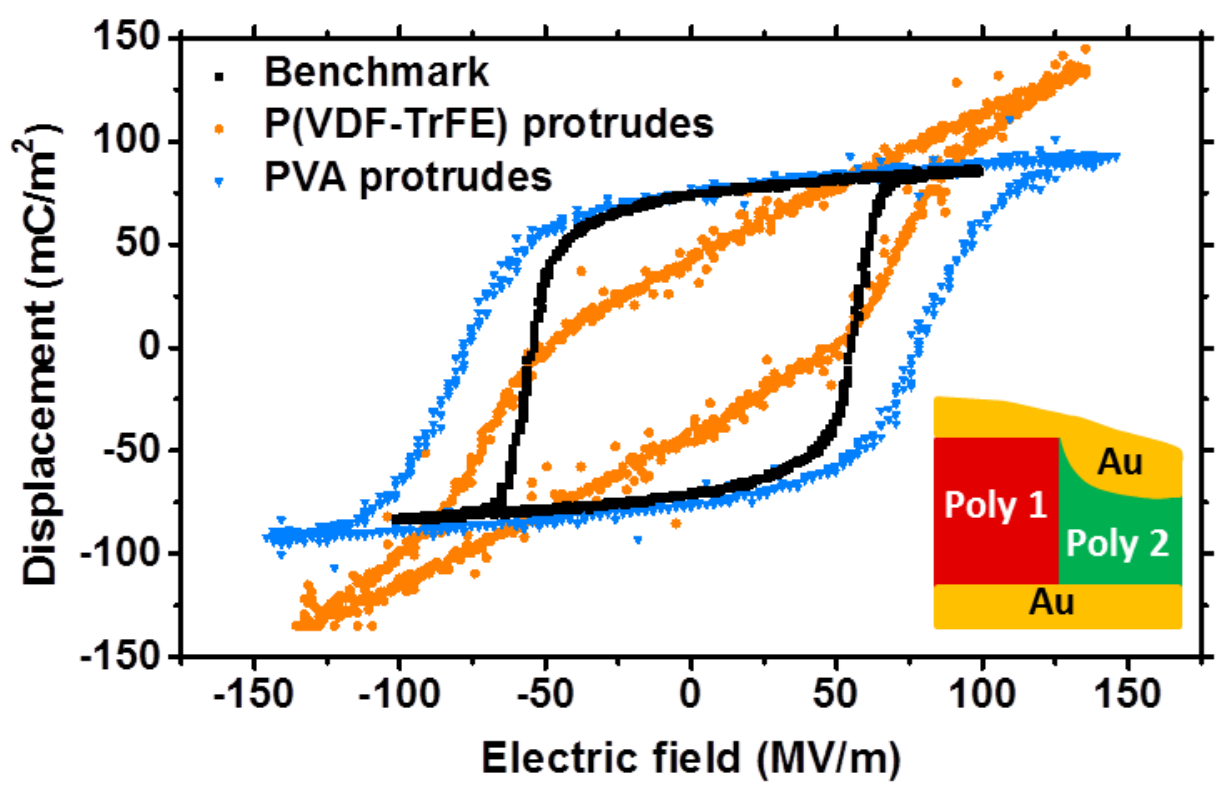


Fig. 6. Typical ferroelectric hysteresis loops. The charge displacement is presented as a function of electric field. The black curve is measured for an unpatterned P(VDF-TrFE) capacitor, which serves as a benchmark. The blue and orange curves are measured for linear arrays. The layout is schematically depicted in the inset. The device area of these crossbar capacitors amounted to $0.1 \mathrm{~mm}^{2}$. The blue curve represents a linear array of a micromolded PVA grating backfilled with $P(V D F-T r F E)$. Here the PVA protrudes. The orange curve represents a linear array of a micromolded P(VDF-TrFE) grating backfilled with PVA. Here the P(VDFTrFE) protrudes. The measurements were performed at $100 \mathrm{~Hz}$ using a series reference capacitor of $220 \mathrm{nF}$.

The measured displacement can depend on the characteristics of the equivalent circuit of the device under test. To ensure that the suppression of remanent polarization, observed for the binary polymer array, where $\mathrm{P}(\mathrm{VDF}-\mathrm{TrFE})$ protrudes, is actual and not a measurement artefact, we performed a detailed analysis, which is presented in the next section.

\section{Remanent polarization of binary linear arrays}

In this section we analyze the electrical behaviour of ferroelectric capacitors comprising a linear array of $\mathrm{P}(\mathrm{VDF}-\mathrm{TrFE})$ and PVA. The equivalent electrical circuit is a linear capacitor of PVA in parallel with the ferroelectric capacitor of $\mathrm{P}(\mathrm{VDF}-\mathrm{TrFE})$. We show that when using a conventional Sawyer-Tower set-up, the apparent polarization can significantly deviate from the actual remanent polarization. Furthermore, we show that by performing shunt measurements, using a reference resistor instead of a reference capacitor, this ambiguity can be eliminated. We experimentally verified that the hysteresis loops of the binary linear arrays, as extracted from the switching current, always yield the actual remanent polarization. For arrays, where the P(VDFTrFE) protrudes, the polarization is incomplete. Based on numerical simulations, we argue that the origin of the incomplete polarization is presumably an inhomogeneous field distribution over the $\mathrm{P}(\mathrm{VDF}-\mathrm{TrFE})$. The calculations also explain why the inverted arrays are similar to the unpatterned, benchmark capacitors. 
Different methods to measure the polarization of a ferroelectric capacitor have been reported. ${ }^{38,39}$ The most commonly used technique is the Sawyer-Tower method which relies on charge measurement from a linear reference capacitor in series with the ferroelectric capacitor. A voltage, $V$, is applied over the electrical circuit and the voltage drop over the reference capacitor, $V_{r e f}$, is measured. With the known value of the reference capacitance, $C_{r e f}$, the density of surface charges, $Q$, at the reference capacitor can be calculated as $Q=C_{r e f} V_{r e f}{ }^{40}$. When the reference capacitance is sufficiently large the voltage drop over $C_{r e f}$ is small, the ferroelectric capacitor is fully polarized and since the two capacitors are in series, $Q=\varepsilon_{0} V_{\text {ferro }} / d+P$, where $d$ is the thickness of the ferroelectric layer, $P$ is the polarization, and $V_{\text {ferro }}$ is the voltage drop over the ferroelectric capacitor. The remanent polarization, $P_{r}$, is therefore approximately the charge density obtained at $V=0$, i.e. $Q_{r}=P_{r}$. We thus call $Q_{r}$ the apparent remanent polarization. Here and below, the subscript, $r$, indicates the condition $V=0$.

In the case of a binary array, we need to add a linear capacitor in parallel to the ferroelectric one, as illustrated in the insets of Fig. 7. The equality $Q_{r}=P_{r}$ is no longer valid. At $V=0$, electroneutrality requires:

$C_{\text {ferro }} V_{\text {ferro }}+P_{r}+C_{\text {parallel }} V_{\text {ferro }}=Q_{r}$

where $Q_{r}=C_{r e f} V_{r e f}=-C_{r e f} V_{\text {ferro }}$. With this, the strict relation between the apparent remanent polarization, $Q_{r}$, and the actual remanent polarization, $P_{r}$, reads:

$Q_{r}=\frac{C_{\text {ref }} P_{r}}{C_{r e f}+C_{\text {parallel }}+C_{\text {ferro }}}$

We see that $Q_{r}=P_{r}$ is recovered when $C_{r e f}$ is much larger than the combined capacitance of $C_{\text {parallel }}$ and $C_{\text {ferro }}$. However, when $C_{\text {ref }}$ is not sufficiently large compared with either $C_{\text {parallel }}$ or $C_{f e r r o}$, the apparent remanent polarization can be significantly smaller than the actual remanent polarization. 


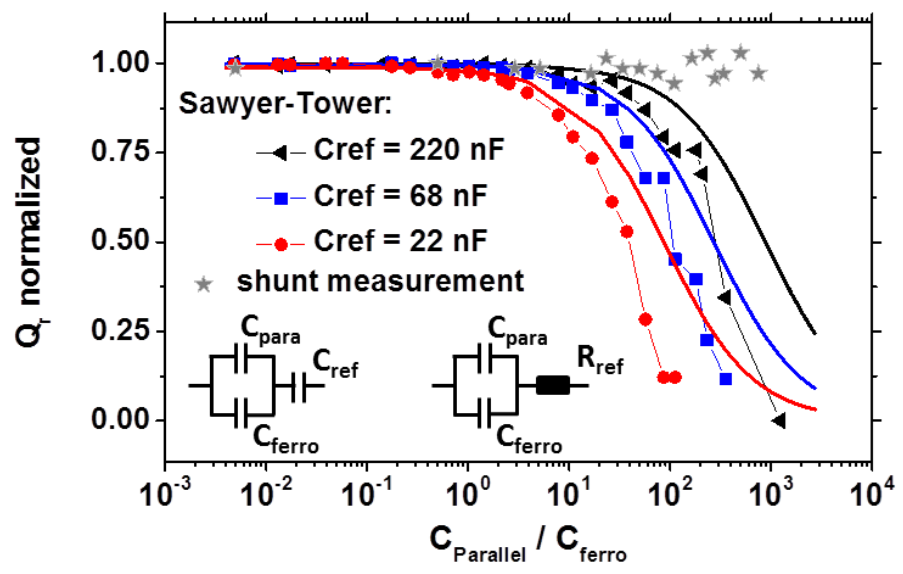

Fig. 7. Apparent polarization for ferroelectric capacitors in parallel with a linear capacitance. Dots represent the apparent remanent polarization as a function of $C_{\text {parallel }} / C_{\text {ferro, }}$ measured with a Sawyer-Tower set-up as shown in the left inset. The values of the parallel and reference capacitance were varied deliberately. The fully drawn curves are calculated according to Eq.[2]. The stars represent the remanent polarization extracted from switching current measurements using a reference shunt resistor, as shown in the right inset.

To experimentally substantiate the difference between apparent- and actual remanent polarization, we connected an unstructured ferroelectric capacitor in parallel with a linear capacitor and measured the polarization with the Sawyer-Tower circuit (left inset of Fig. 7). We deliberately varied the values of the capacitances. The measured apparent polarization is presented as a function of $C_{\text {parallel }} / C_{\text {ferro }}$ in Fig. 7 . The fully drawn lines are calculated according to Eq. [2]. A good agreement is obtained. Fig. 7 clearly shows that, depending on the device layout and value of the reference capacitance, the apparent polarization, as measured with a Sawyer-Tower circuit, can significantly deviate from the actual remanent polarization.

This apparent incomplete polarization can be circumvented by using shunt measurements. The reference capacitor is replaced by a resistor and the switching current is calculated from the voltage drop over the shunt resistance. The equivalent electrical circuit is shown in the right inset of Fig. 7. The hysteresis loop is then obtained by integrating the measured switching current. The stars in Fig. 7 represent the polarization as extracted from switching current measurements. 
Artefacts of the conventional Sawyer-Tower configuration are eliminated, as the remanent polarization no longer depends on the ratio of $C_{\text {parallel }} / C_{f e r r o}$.

In order to eliminate measurement artefacts we analyzed the patterned capacitors using both Sawyer-Tower and shunt measurements. Both methods showed similar hysteresis loops. Extracted parameters are presented in Table 1, each value averaged over 5 different capacitors. The linear capacitance was independently extracted from impedance measurements. Table 1 demonstrates that capacitors of binary arrays, where $\mathrm{P}(\mathrm{VDF}-\mathrm{TrFE})$ protrudes, show incomplete polarization. Capacitors in the inverted arrays, where PVA protrudes, are comparable to the unpatterned, benchmark capacitors.

Table 1. Extracted parameters for linear arrays of P(VDF-TrFE) and PVA. Each value is the mean average of 5 capacitors. The remanent polarization is extracted from Sawyer-Tower measurements using either a reference capacitor or a reference shunt resistor. The linear capacitance is extracted both from the slope of the hysteresis loops at zero bias and from small angle impedance measurements. The geometric capacitance as calculated from the measured topography is included for comparison.

\begin{tabular}{|l|c|c|}
\hline \multicolumn{1}{|c|}{ Sample } & P(VDF-TrFE) protrudes & PVA protrudes \\
\hline Polarization from: & $42.6 \pm 4.4$ & $70.8 \pm 8.0$ \\
\hline Sawyer-Tower $\left[\mathrm{mC} / \mathrm{m}^{2}\right]$ & $41.2 \pm 4.7$ & $70.2 \pm 8.9$ \\
\hline Shunt measurement $\left[\mathrm{mC} / \mathrm{m}^{2}\right]$ & 5.02 & 10.3 \\
\hline Capacitance from: & $17.4 \pm 5.6$ & $13.6 \pm 1.2$ \\
\hline Geometry [pF] & $16.2 \pm 4.9$ & $12.8 \pm 2.4$ \\
\hline Hysteresis loop $[\mathrm{pF}]$ & &
\end{tabular}

To explain the experimentally observed incomplete polarization measured for the binary array in which P(VDF-TrFE) protrudes, we calculated the electric field distribution. We take a 2D grid with mirror plane boundary conditions with the experimental geometry as shown in the inset of Fig. 8. The dielectric constants of the PVA and P(VDF-TrFE) are taken as 3 and 10, respectively. The potential on the electrodes is fixed. The electric field is then calculated using Poisson's equation. Since, for simplicity, the ferroelectric dipoles in the P(VDF-TrFE) layer are neglected, 
the simulated field distribution is realistic only at low electric fields, much lower than the coercive field. Fig. 8 shows the calculated electric field lines. The electric field over the P(VDFTrFE) lines is inhomogeneous. The field is high in the center and low at the edges. The large region of reduced electric field in the $\mathrm{P}(\mathrm{VDF}-\mathrm{TrFE})$ at the interface with the PVA can be interpreted as a reduction in the effective device area, which explains the measured incomplete polarization. We note that the simulations for the inverted linear arrays, where PVA protrudes, corroborate the full polarization. Therefore, the hysteresis loops of these capacitors are comparable to those of the unpatterned, benchmark capacitors.

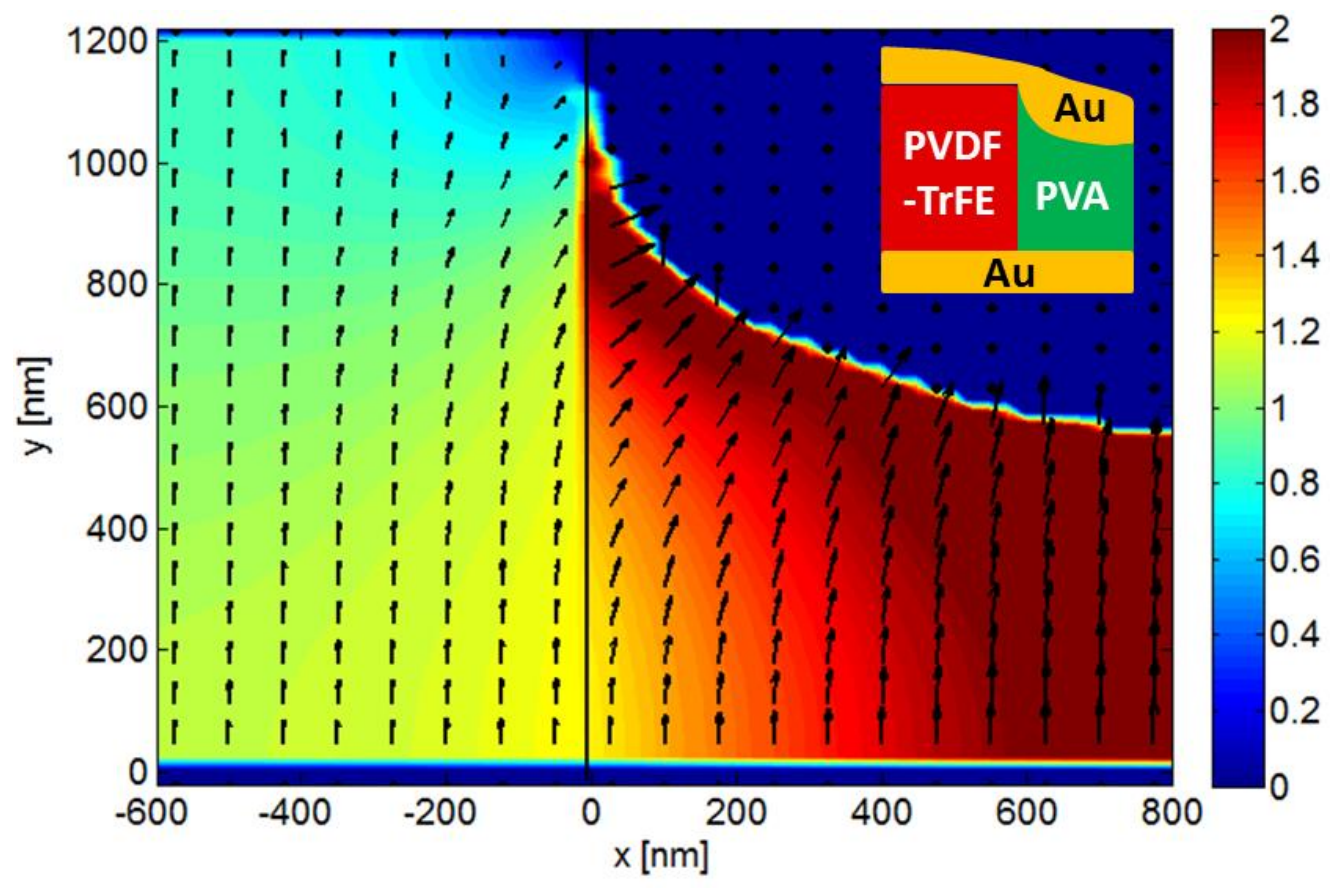

Fig. 8. Electric field distribution. The electric field calculated for a $2 D$ grid around the $P(V D F-$ TrFE)/PVA interface with mirror plane boundary conditions. The insets depicts the experimental capacitor geometry. $P(V D F-T r F E)$ protrudes the binary linear array. The color scale represents the electric field normalized to V/d max with $d_{\max }$ the P(VDF-TrFE) thickness.' 


\section{Summary and conclusion}

Line gratings of the ferroelectric polymer $\mathrm{P}(\mathrm{VDF}-\mathrm{TrFE})$ were fabricated over large area by solution micromolding using a PDMS stamp. To fabricate capacitors the grating was backfilled with the electrically insulating polymer PVA, which was chosen because it dissolves in deionized water, a solvent that is orthogonal to $\mathrm{P}(\mathrm{VDF}-\mathrm{TrFE})$ and does not swell the stamp. Inverted arrays by micromolding PVA and backfilling with P(VDF-TrFE) were fabricated as well. Capacitors were finished in crossbar geometry, and for the first time we analyzed the device physics. The equivalent electrical circuit is a linear capacitor of PVA in parallel with the ferroelectric capacitor of P(VDF-TrFE). The consequences for conventional Sawyer-Tower measurements are analyzed and experimentally verified. The apparent polarization can significantly deviate from the actual remanent polarization. We show that from shunt measurements, using a reference resistor instead of a reference capacitor, this ambiguity can be eliminated. We experimentally verified that the hysteresis loops of the linear arrays extracted from the switching current always yield the actual remanent polarization.

The arrays exhibit topography as the height of the backfilled polymer is always lower than that of the initially micromolded grating. In capacitors made from binary arrays, where the P(VDFTrFE) protrudes, the polarization is incomplete. Numerical simulations of the electric field distribution show that the origin is a decrease in effective electrode area. On the other hand, in binary arrays, where PVA protrudes, the ferroelectric hysteresis loops are similar to those of unpatterned, benchmark capacitors, which suggests that solution micromolding is a versatile technology to study confinement by scaling down the lateral dimensions of the grating.

\section{Acknowledgements}

We gratefully acknowledge technical support from C. Bauer and F. Keller from the Max Plank Institute for Polymer Research, Mainz, Germany. We are indebted to H. Wondergem, Philips Research, Eindhoven, The Netherlands and M. Steiert from the Max Plank Institute for Polymer Research for X-ray analysis of $\mathrm{P}(\mathrm{VDF}-\mathrm{TrFE})$ films and gratings. We acknowledge financial 
support from the Max Planck Institute for Polymer Research and from KAU by project 71-10035-HiCi. Author K. A. acknowledges the Alexander von Humboldt Foundation for the funding provided in the framework of the Sofja Kovalevskaja Award, endowed by the Federal Ministry of Education and Research, Germany. Author T. L. acknowledges financial support by the Graduate School Materials Science in Mainz.

\section{References}

1 R. C. G. Naber, K. Asadi, P. W. M. Blom, D. M. de Leeuw, and B. de Boer, Advanced Materials 22 (9), 933 (2010).

2 T. Sekitani, K. Zaitsu, Y. Noguchi, K. Ishibe, M. Takamiya, T. Sakurai, and T. Someya, IEEE Transactions on Electron Devices 56 (5), 1027 (2009).

3 S. Zhang, Z. Liang, Q. Wang, and Q. M. Zhang, presented at the Materials Research Society Symposium Proceedings 889, 167-186 (2006).

A. J. J. M. van Breemen, J. B. P. H. van der Putten, R. Cai, K. Reimann, A. W. Marsman, N. Willard, D. M. de Leeuw, and G. H. Gelinck, Applied Physics Letters 98 (18), 183302 (2011).

5 S. J. Kang, Y. J. Park, J. Y. Hwang, H. J. Jeong, J. S. Lee, K. J. Kim, H. C. Kim, J. Huh, and C. Park, Advanced Materials 19 (4), 581 (2007).

6 Y. J. Park, S. J. Kang, Y. Shin, R. H. Kim, I. Bae, and C. Park, Current Applied Physics 11 (2, Supplement), e30 (2011).

D. E. Martínez-Tong, M. Soccio, M. C. García-Gutiérrez, A. Nogales, D. R. Rueda, N. Alayo, F. Pérez-Murano, and T. A. Ezquerra, Applied Physics Letters 102 (19), 191601 (2013).

Z. Hu, M. Tian, B. Nysten, and A. M. Jonas, Nature Materials 8 (1), 62 (2009).

X.-Z. Chen, X. Chen, X. Guo, Y.-S. Cui, Q.-D. Shen, H.-X. Ge, Nanoscale 2014, 6 (22), 13945. Xunlin Qiu, Journal of Applied Physics 108 (1) 011101 (2010).

Y. Xia and G. M. Whitesides, Angewandte Chemie International Edition 37 (5), 550 (1998).

E. Kim, Y. Xia, and G. M. Whitesides, Nature 376 (6541), 581 (1995).

W. Ong, C. Ke, P. Lim, A. Kumar, K. Zeng, and G. W. Ho, Polymer (United Kingdom) 54 (20), $5330(2013)$. 
Y. J. Park, Y. S. Kang, and C. Park, European Polymer Journal 41 (5), 1002 (2005).

J. L. Lutkenhaus, K. McEnnis, A. Serghei, and T. P. Russell, Macromolecules 43 (8), 3844 (2010).

H. G. Kassa, L. Nougaret, R. Cai, A. Marrani, B. Nysten, Z. Hu, and A. M. Jonas, Macromolecules 47 (14), 4711 (2014).

G. Canavese, S. Stassi, V. Cauda, A. Verna, P. Motto, A. Chiodoni, S. L. Marasso, and D. Demarchi, IEEE Sensors Journal 13 (6), 2237 (2013).

V. Cauda, S. Stassi, K. Bejtka, and G. Canavese, ACS Applied Materials \& Interfaces 5 (13), $6430(2013)$.

D. Guo and N. Setter, Macromolecules, 461883 (2013)

M. Li, I. Katsouras, K. Asadi, P.W.M. Blom and D.M. de Leeuw Applied Physics Letters, 103, 072903 (2013)

V. Cauda, B. Torre, A. Falqui, G. Canavese, S. Stassi, T. Bein, and M. Pizzi, Chemistry of Materials 24 (21), 4215 (2012).

S. J. Kang, I. Bae, Y. J. Shin, Y. J. Park, J. Huh, S.-M Park, H.-C. Kim, and C. Park, Nano Letters $11(1), 138$ (2010).

K. Asadi, D. M. de Leeuw, B. de Boer, and P. W. M. Blom, Nature Materials 7 (7), 547 (2008).

K. Asadi, M. Li, P. W. M. Blom, M. Kemerink, and D. M. de Leeuw, Materials Today 14 (12), $592(2011)$.

A. J. J. M. Van Breemen, J. L. Van Der Steen, G. Van Heck, R. Wang, V. Khikhlovskyi, M. Kemerink, and G. H. Gelinck, Applied Physics Express 7 (3), 031602 (2014).

M. A. Khan, U. S. Bhansali, D. Cha, H. N. Alshareef, Advanced Functional Materials 2013, 23 (17), 2145-2152.

M. Kemerink, K. Asadi, P. W. M. Blom, and D. M. de Leeuw, Organic Electronics 13 (1), 147 (2012).

L. Nougaret, H. G. Kassa, R. Cai, T. Patois, B. Nysten, A. J. J. M. van Breemen, G. H. Gelinck, D. M. de Leeuw, A. Marrani, Z. Hu, and A. M. Jonas, ACS Nano 8 (4), 3498 (2014).

S. H. Sung, B. W. Boudouris, ACS Macro Letters 2015, 4, 293

A. J. J. M. van Breemen, T. Zaba, V. Khikhlovskyi, J. J. Michels, R. A. J. Janssen, M. Kemerink, G. H. Gelinck, Advanced Functional Materials 2015, 25 (2), 278. 
31 V. Khikhlovskyi, R. Wang, A. J. J. M. van Breemen, G. H. Gelinck, R. A. J. Janssen, M. Kemerink, The Journal of Physical Chemistry C 2014, 118 (6), 3305-3312.

32 Y. Li, C. Liu, L. Pan, L. Pu, H. Sirringhaus, Y. Shi, Applied Physics Letters 2011, 98 (17), 173306.

33 J. N. Lee, C. Park, and G. M. Whitesides, Analytical Chemistry 75 (23), 6544 (2003).

34 H. S. Nalwa, Ferroelectric Polymers: Chemistry, Physics, and Applications. (Marcel Dekker, New York, 1995).

35 T. Furukawa, Phase Transitions 18 (3-4), 143 (1989).

36 D. Guo, I. Slolichnov and N. Setter, The Journal of Physical Chemistry B, 115, 13455 (2011).

37 T. Feng, D. Xie, Y. Zang, X. Wu, T. Ren, W. Pan, Integrated Ferroelectrics (2013), 141, 187.

38 M. G. Cain, Characterisation of Ferroelectric Thin Films: Solutions for Metrology. (Springer, 2014).

39 K. Prume, Th. Schmitz, and S. Tiedke, in Polar Oxides (Wiley-VCH Verlag GmbH \& Co. KGaA, 2005), pp. 53.

40 C. T. Black, C. Farrell, and T. J. Licata, Applied Physics Letters 71 (14), 2041 (1997). 